\title{
Randomized Effectiveness Trial of a Brief Course of Acupuncture for Posttraumatic Stress Disorder
}

\author{
Charles C. Engel, MD, MPH, $+\ddagger$ Elizabeth H. Cordova, MA, * David M. Benedek, MD, $\dagger$ \\ Xian Liu, PhD, * $+\ddagger$ Kristie L. Gore, PhD, * $+\ddagger$ Christine Goertz, DC, PhD, $\S$ Michael C. Freed, PhD, * $\dagger$ \\ Cindy Crawford, BA, || Wayne B. Jonas, MD, || and Robert J. Ursano, MD†t
}

Background: Initial posttraumatic stress disorder (PTSD) care is often delayed and many with PTSD go untreated. Acupuncture appears to be a safe, potentially nonstigmatizing treatment that reduces symptoms of anxiety, depression, and chronic pain, but little is known about its effect on PTSD.

Methods: Fifty-five service members meeting research diagnostic criteria for PTSD were randomized to usual PTSD care (UPC) plus eight 60-minute sessions of acupuncture conducted twice weekly or to UPC alone. Outcomes were assessed at baseline and 4, 8, and 12 weeks postrandomization. The primary study outcomes were difference in PTSD symptom improvement on the PTSD Checklist (PCL) and the Clinician-administered PTSD Scale (CAPS) from baseline to 12-week follow-up between the 2 treatment groups. Secondary outcomes were depression, pain severity, and mental and physical health functioning. Mixed model regression and $t$ test analyses were applied to the data.

Results: Mean improvement in PTSD severity was significantly greater among those receiving acupuncture than in those receiving UPC (PCL $\Delta=19.8 \pm 13.3$ vs. $9.7 \pm 12.9, P<0.001$; CAPS $\Delta=35.0$ \pm 20.26 vs. $10.9 \pm 20.8, P<0.0001)$. Acupuncture was also associated with significantly greater improvements in depression, pain, and physical and mental health functioning. Pre-post effect-sizes for these outcomes were large and robust.

From the *Deployment Health Clinical Center, Walter Reed National Military Medical Center; †Center for the Study of Traumatic Stress (CSTS); $₫$ Department of Psychiatry, Uniformed Services University of the Health Sciences, Bethesda, MD; §Palmer Center for Chiropractic Research, Davenport, IA; and $\|$ Samueli Institute, Alexandria, VA.

Supported by the Uniformed Services University of the Health Sciences (USUHS) under Award No. MDA905-03-C-0003.

The views, opinions, and/or findings contained in this report are those of the author(s) and should not be construed as an official Department of the Defense position, policy, or decision unless so designated by other documentation.

Papers based on the results of this research were presented at The International Society of Traumatic Stress Studies Annual Meeting, November 13-15, 2008, Chicago, IL, and the 11th Annual Force Health Protection Conference, August 11-14, 2008, Albuquerque, NM. Posters were presented at the 60th Institute on Psychiatric Services, October 2-5, 2008, Chicago, IL, and the Uniformed Services University of the Health Sciences Research Week, May 2009, Bethesda, MD.

The authors declare no conflict of interest.

Reprints: Charles C. Engel, MD, MPH, Department of Psychiatry, School of Medicine, Uniformed Services University of the Health Sciences, 4301 Jones Bridge Rd, Bethesda, MD 20814. E-mail: cengel@rand.org.

Copyright (C) 2014 by Lippincott Williams \& Wilkins

ISSN: 0025-7079/14/5212-0S57
Conclusions: Acupuncture was effective for reducing PTSD symptoms. Limitations included small sample size and inability to parse specific treatment mechanisms. Larger multisite trials with longer follow-up, comparisons to standard PTSD treatments, and assessments of treatment acceptability are needed. Acupuncture is a novel therapeutic option that may help to improve population reach of PTSD treatment.

Key Words: acupuncture, PTSD, CAM, military, primary care, mental health

(Med Care 2014;52: S57-S64)

$P$ osttraumatic stress disorder (PTSD) is highly prevalent and disabling, particularly after military service in a war zone. Recent studies in the US military have identified PTSD in up to $17 \%$ after combat deployment and $9 \%$ in military primary care settings. ${ }^{1,2}$ Although FDA-approved medications and empirically valid psychotherapies for PTSD treatment exist, there is sparse evidence of effectiveness in active military samples. ${ }^{3}$ What evidence exists is indirect, gleaned mainly from US Veterans Affairs settings involving older patients and chronic PTSD, and suggests smaller treatment effects for war-related PTSD. ${ }^{4-6}$

Individuals needing PTSD treatment often do not receive it. Hoge et $\mathrm{al}^{2}$ examined recently returned Iraq and Afghanistan War veterans with PTSD, generalized anxiety disorder, or major depression, and found that $81 \%$ acknowledged having a problem, whereas only $41 \%$ reported interest in receiving help. Only $30 \%$ and $14 \%$ reported professional help or mental health specialist care in the past year, respectively. Furthermore, they found that $25 \%$ of affected service members believed mental health treatment did not work, $28 \%$ did not trust mental health providers, and $41 \%$ thought treatment would be embarrassing. ${ }^{2}$

Similar challenges seem to exist in nonmilitary settings. Wang et $\mathrm{al}^{7}$ found that only $7 \%$ of a representative general population sample with PTSD reported initial treatment contact in the first year of illness and the median treatment delay was 12 years. The National Comorbidity Survey found that among nonpatients with PTSD and selfperceived need for assistance, $59 \%$ of men and $26 \%$ of women believed that PTSD treatment would not help. ${ }^{8}$ These findings suggest that a novel and effective treatment option may help increase the proportion of those with PTSD who seek early treatment. 
Acupuncture is an increasingly popular nontraditional treatment approach that seems to be safe and effective for anxiety, depression, stress, and chronic pain, symptoms that often accompany PTSD. ${ }^{9-13}$ The neurological mechanisms by which acupuncture effects anxiety, pain, and depression are not entirely understood. However, neuroimaging and neurophysiological studies have found that acupuncture has limbic system, prefrontal cortex, and autonomic nervous system effects. ${ }^{14-16}$ Many of these neural systems have been implicated in the development and maintenance of anxiety symptoms to include symptoms of PTSD. ${ }^{17-19}$ For example, the endogenous opioid system may mediate sympathoinhibitory acupuncture effects, ${ }^{20,21}$ whereas acupuncture needle location ${ }^{14,22}$ and type ${ }^{15,16,23}$ stimulate discrete and differential neural responses. If effective for PTSD, acupuncture could hold promise as an acceptable treatment alternative. Remarkably, the only published high-quality randomized trial evaluating manual acupuncture for the treatment of PTSD $^{24}$ found acupuncture was comparable with group cognitive-behavioral therapy and superior to waitlist controls. ${ }^{25}$ The only other published controlled clinical trials used electroacupuncture protocols and, while suggesting positive outcomes, offer inadequate descriptions of key methodological details (eg, no data on completeness and duration of follow-up). ${ }^{26-28}$ The present study is the first to evaluate the use of a brief course of manual acupuncture for military-related PTSD.

The hypothesis of the present trial was that a brief, 4-week course of acupuncture (eight 60 min treatments) in combination with patients' usual PTSD care (UPC) would be significantly more effective than UPC alone for active duty military personnel with PTSD. The primary outcome was PTSD symptom severity over 12 weeks of follow-up. Other outcomes were depression, pain, and mental and physical health functioning. An effectiveness design with a UPC control condition was chosen for 2 main reasons: (1) the goal was to assess the added benefit of brief acupuncture combined with patients' usual PTSD treatment over the benefits of usual and customary PTSD treatment alone; and (2) a comparison of acupuncture to sham requires strict participant selection, reducing generalizability of findings to the population of interest, in this case active military personnel obtaining care for PTSD. In addition, it is unclear at this point what the most appropriate type of sham for use in acupuncture studies is. ${ }^{29}$

\section{METHODS}

The design was a 2-parallel arm, randomized controlled trial comparing the effectiveness of semistructured, brief adjunctive acupuncture for patients receiving UPC to the effectiveness of UPC alone. Acupuncture was delivered in 8 sessions over 4 weeks. Participants were assessed at baseline and at 4,8 , and 12 weeks postrandomization. After completion of study follow-up, participants in the UPC group were offered the study acupuncture intervention and a list of local mental health services.

Participants were active duty military personnel enrolled between February 2006 and June 2007. Most participants were recruited from primary care clinics at Walter Reed Army
Medical Center (WRAMC) (68\%), whereas self-referrals from advertisements at WRAMC (19\%) and referrals from providers and patients (13\%) made up the remainder of the sample. To meet inclusion criteria, active duty candidates between the ages of 18 and 65 years old had to score $\geq 30$ on the PTSD Checklist (PCL) $)^{30}$ and meet criteria for PTSD on the Clinicianadministered PTSD Scale (CAPS) using the 1-2 scoring rule. ${ }^{31}$ Individuals were excluded for: (1) $\geq 1$ PTSD treatment changes (ie, medication or psychotherapy stop/start, clinically significant change in therapy frequency or medication dosing) in the past 8 weeks; (2) average self-reported pain $\geq 8$ on the Numeric Rating Scale for $\mathrm{Pain}^{32}$; (3) any acupuncture treatment in the past 6 months; (4) pregnancy; (5) moderate or severe TBI; (6) psychosis in the past 2 years; (7) serious instability in medical or psychiatric status based on principal investigator or medical monitor judgment; and (8) any DSMIV criterion A traumatic experience in the past 30 days. After description of the study to participants, written informed consent was obtained per WRAMC IRB requirements.

Participants were randomized to either acupuncture or UPC. Those assigned to acupuncture were randomly assigned 1 of the 3 study acupuncturists delivering treatment to avoid selective assignment to any 1 acupuncturist. An independent acupuncturist who did not treat participants and a psychologist performed the assessments, and both were blinded to treatment assignment. A computer was used to generate random assignments and study ID numbers. Following consent, a research team member blinded to treatment assignment conveyed the group assignment to the study coordinator, who notified the participant of the result. The master list, consent forms, and deidentified participant data were secured separately under double lock and key. Only the study coordinator had access to these locked files. Acupuncture and study assessments occurred in private offices at the Walter Reed Social Work Clinic.

\section{Acupuncture Treatment}

Our goal was to test an easily implementable course of acupuncture treatment that might eventually result in wide clinical use as an adjunct to UPC across a variety of settings. The briefest potentially safe and effective treatment course was therefore sought. Electroacupuncture, for example, was considered but rejected due to potential for safety concerns that might prevent implementation in some settings.

Acupuncturists were licensed, practicing regularly, and recipients of advanced degrees (M.Ac.) from the Tai Sophia Institute for the Healing Arts, a program emphasizing traditional Chinese medicine philosophies and using 5 elements theory as overall guide to treatment. These theories hold that bodily channels (meridians) of energy flow (Qi) influence and maintain an individual's health. Illness is viewed as the result of disharmonious, imbalanced, or blocked Qi within the body and treatment from the Chinese medicine perspective targets certain patterns of disharmony that are detected by taking a history and examining the patient's pulse, tongue, eyes, and other characteristics.

Three acupuncturists and 2 study research investigators (C.G. and W.B.J.) collaborated in a series of several meetings to codify procedures for brief acupuncture. The result was an 
intervention manual to maximize treatment replicability. To balance replicability and need for individualized treatment tailoring, the first 4 sessions were standardized for all participants, and the last 4 sessions allowed acupuncturists flexibility to individualize based on standard diagnostic criteria (pulse, tongue, symptoms, color, odor, etc). The first 4 standard treatments each used a unique point prescription to facilitate clearing of trauma, calming the spirit, and balancing Qi. Each of the following acupoints were used at least once: Urinary Bladder 13, 14, 15, 18, 20, 23; Liver 3; Large Intestine 4; Heart 5, 7; Pericardium 6; Kidney 3, 9; Ren 4, 15; $\mathrm{Du}$ 24; Ear Shenmen; and Yintang.

All acupuncturists used hair-thin, solid needles (Seirin brand, $\mathrm{J}$ type: $0.14,0.16$, and $0.2 \mathrm{~mm}$ and $\mathrm{L}$ type: $0.2 \mathrm{~mm}$ ) that were manually inserted into subcutaneous tissue or muscle at specified points for a set period of 15-30 minutes depending on point prescription, typical for outpatient settings. Standard sanitation procedures were followed: needles were new and obtained from individually sealed packages, acupuncturists followed proper hand-washing procedures and wore sterile rubber gloves, needles sites were rubbed with antibacterial wipes, and used needles were disposed of in designated sharps containers to prevent contamination.

Total length of treatment sessions was approximately 60 minutes to allow for diagnostic evaluation by the acupuncturist before each treatment. Treatments occurred twice per week for 4 weeks (total of 8 treatments). Before data collection, acupuncturists calibrated their technique on actors portraying symptoms and engaged in discussion to identify and resolve differing interpretations of symptoms, treatment manual instructions, and manual adherence to ensure treatment reliability.

\section{Usual PTSD Treatment}

All participants received UPC at WRAMC. Care was guided by existing standards, outlined in the VA/DoD Clinical Practice Guideline for Management of Post-Traumatic Stress. ${ }^{33}$ Psychotherapies for PTSD typically contain an eclectic mix of approaches with an emphasis on elements of empirically validated PTSD treatments such as prolonged exposure and nontrauma-focused cognitive-behavioral therapy. Medications and psychotherapy were tracked in patients in both intervention arms, but no effort was made to maximize the fidelity of usual care modalities that study patients received. Common medications used for patients with PTSD were antidepressants, most often selective serotonin reuptake inhibitors, and prazosin, a centrally acting $\alpha-1$ receptor antagonist shown to decrease trauma-related nightmares and secondarily improve sleep and global clinical status. ${ }^{34}$ Pharmacotherapies are often selected to target common comorbid symptoms (eg, insomnia, depression, panic, and chronic pain). These may involve anxiolytics/sedativehypnotics, sedating antidepressants, mood stabilizers, anticonvulsants, and analgesics. Study participants in both groups were asked to refrain during the study from engaging in other complementary and alternative treatments, including acupuncture outside the study intervention. Study participants tracked medications and other treatments in a daily diary and reported on treatment utilization at each follow-up.

\section{Primary Outcomes}

The PCL, Civilian Version, ${ }^{30}$ was used as the primary outcome measure to assess PTSD symptom severity at baseline and at the 3 follow-ups. It is a 17-item measure that assesses the symptoms of PTSD in the DSM-IV and yields a total score ranging from 17 to 85 . The civilian rather than the military version was used so that participants could report all PTSD symptoms, regardless of whether a military trauma had initiated them. The CAPS ${ }^{31}$ is the consensus diagnostic gold standard for PTSD. ${ }^{35}$ The CAPS Total Severity Score was used to estimate changes in PTSD symptom severity from baseline to 12-week follow-up. CAPS raters were blinded psychologists who received training in CAPS administration and scoring, and participated in reliability checks versus other CAPS raters before study initiation.

\section{Secondary Outcomes}

The Numeric Rating Scale (NRS) for Pain $^{32}$ was used to assess pain at baseline and all follow-up assessments. The NRS is an 11-point scale that allows individuals to rate their average pain intensity over the past week from 0 (no pain) to 10 (pain as bad as you can imagine).

The Beck Depression Inventory-II (BDI-II) ${ }^{36}$ was used to measure severity of depression symptoms at baseline and all follow-up assessments. The BDI-II is a psychometrically sound 21-item self-report measure, scored 0-63. Scores $<15$ indicate mild or no depression symptoms, 15-30 indicate moderate depression symptoms, and $>30$ indicates severe depression symptoms.

The SF-36-Revised ${ }^{37}$ was used at baseline and 12week follow-up to evaluate the physical (PCS) and mental (MCS) component summary measures of health functioning.

\section{Treatment Fidelity}

All acupuncture sessions were audio-recorded and acupuncturists completed an 18-item questionnaire after each treatment session that captured self-reported fidelity to the manualized treatment for that session. Acupuncturist selfassessments of treatment fidelity were consistently high. These steps were taken to serve as regular acupuncturist reminders of the key acupuncture components as they had defined them and to encourage acceptable treatment fidelity.

\section{Statistical Analysis}

Two-tailed $t$ tests analyzed the changing pattern over time of the 2 primary and 4 secondary outcomes for 3 prepost pairs (baseline vs. $4 \mathrm{wk}$ follow-up, baseline vs. $8 \mathrm{wk}$ follow-up, and baseline vs. 12 wk follow-up). All degrees of freedom $(d f)$ for $t$ tests were based on Multiple Imputation. Mixed model regression was used for the primary intent-totreat analysis. Intention-to-treat analysis requires that all randomized participants are included and analyzed according to original treatment assignment. Mixed model regression offers robust handling of missing follow-up data ${ }^{38}$ and addresses the autoregressive error structure of longitudinal data. A prespecified residual variance/covariance structure was used to account for the between-persons random effects. Missing data were imputed using the Markov Chain Monte Carlo Method because of small sample size. ${ }^{39}$ Other than 
imputed means and SDs for $t$ tests, Robey method ${ }^{40}$ was used to calculate the effect size (Cohen $d$ ) and the associated $95 \%$ confidence interval for each pre-post pair.

\section{RESULTS}

\section{Sample Characteristics}

Fifty-five participants were randomized, 28 to UPC plus brief acupuncture and 27 to UPC alone. Table 1 shows that randomization resulted in a comparable distribution of baseline characteristics across the intervention groups with no statistically significant $(P>0.1)$ differences observed between the 2 groups. Sixty percent of participants were receiving psychotropic medications, active counseling, or both at the time of study enrollment. By study's end, the percentage had increased to $73 \%$ and there were no significant differences in treatment change across groups (Table 2). No study-related adverse events were reported or observed.

A total of $78 \%$ participants $(43 / 55)$ completed the full protocol. There were no statistically significant differences in baseline characteristics between trial completers and noncompleters. Figure 1 summarizes participant recruitment, eligibility, enrollment, randomization, dropouts, and follow-up.

\section{PTSD Outcomes}

Statistically significant differences favoring acupuncture were found between the 2 treatment groups for the primary outcome, PCL $\left(\chi_{3}^{2}=97.8, P<0.0001\right)$, and for the CAPS $\left(\chi_{3}^{2}=9.2, P<0.05\right)$. Mixed model least square means were all statistically significant and demonstrated a steeper decline in both PCL and CAPS scores in the acupuncture with UPC group versus the UPC-alone group (Fig. 2).

Table 3 summarizes key trial outcomes using $t$ test analyses. Mean PCL score significantly decreased in the acupuncture with UPC group at 4 weeks (58.1 \pm 11.4 vs. $\left.38.8 \pm 11.6 ; t_{241}=13.4, P<0.0001\right)$. Reductions were maintained at 8 weeks $\left(37.8 \pm 15.0 ; t_{153}=11.0, P<0.0001\right)$ and 12 weeks $\left(37.7 \pm 15.9 ; t_{158}=10.5, P<0.0001\right)$. In contrast, among those in UPC alone, the PCL scores dropped just 4 points from baseline to 4 weeks $\left(55.4 \pm 12.0\right.$ vs. $1.5 \pm 12.2 ; t_{268}=2.7$, $P<0.01$ ), and exhibited a much less steep and dramatic decline compared with the acupuncture with UPC group at 8 weeks $\left(53.8 \pm 14.7 ; t_{219}=0.9, P=0.35\right)$ and 12 weeks $(45.8 \pm 13.9$; $\left.t_{237}=6.0, P<0.001\right)$. CAPS scores showed a similar pattern. Pre-post effect sizes for the change in PCL and CAPS score in the acupuncture with UPC group were consistently very large. ${ }^{32}$

\section{Secondary Outcomes}

The mixed model statistics for the 4 secondary outcome variables were consistent with those for the primary outcome variables. All 4 models showed statistically significant differences between the groups' BDI-II $\left(\chi_{3}^{2}=101.9, P<0.0001\right)$, NRS $\quad\left(\chi_{3}^{2}=137.3, \quad P<0.0001\right), \quad$ SF-36 PCS $\quad\left(\chi_{3}^{2}=46.8\right.$, $P<0.0001)$, and SF-36 MCS $\left(\chi_{3}^{2}=16.7, P<0.0001\right)$ scores.

TABLE 1. Baseline Sample Characteristics by Randomly Allocated Intervention Group*

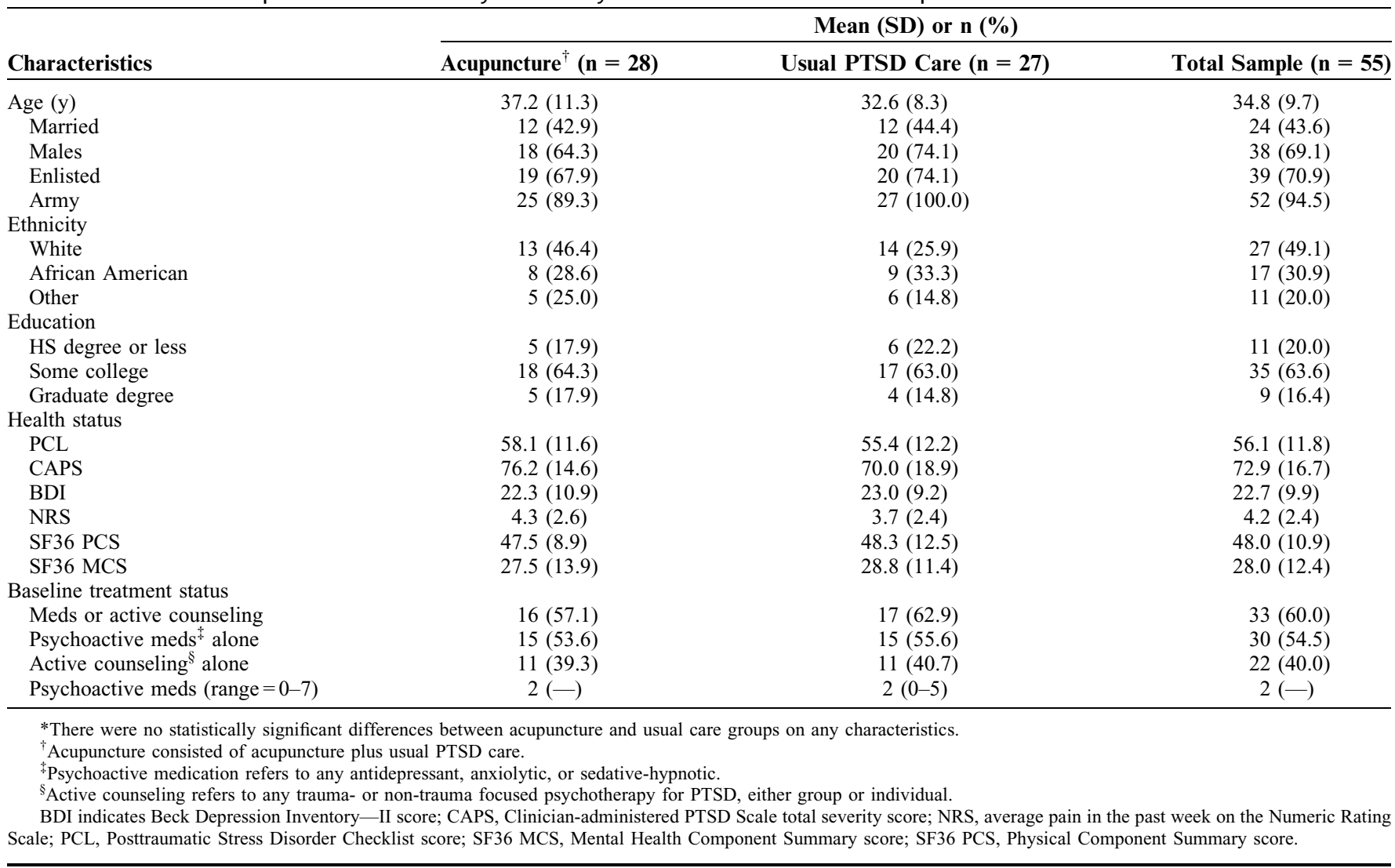


TABLE 2. Participant Treatment Utilization During the Study Follow-up Period

$$
\text { n }(\%)
$$

Treatment During Follow-up

Acupuncture (n $=28)$

Usual PTSD Care $(n=27)$

Total Sample $(\mathrm{n}=\mathbf{5 5})$

Med start or increase

Med stop or decrease

4 (14.3)

1 (3.6)

5 (18.5)

2 (7.4)

9 (16.4)

Counseling start

2 (7.1)

1 (3.7)

Counseling stop

$0(0.0)$

$0(0.0)$

$3(5.5)$

Total treatment changes*

$7(25.0)$

8 (29.6)

$3(5.5)$

$0(0.0)$

$15^{\dagger}(27.3)$

*Treatment change refers to changes in psychoactive medication or active counseling.

${ }^{\dagger} 15$ treatment changes in 13 participants.

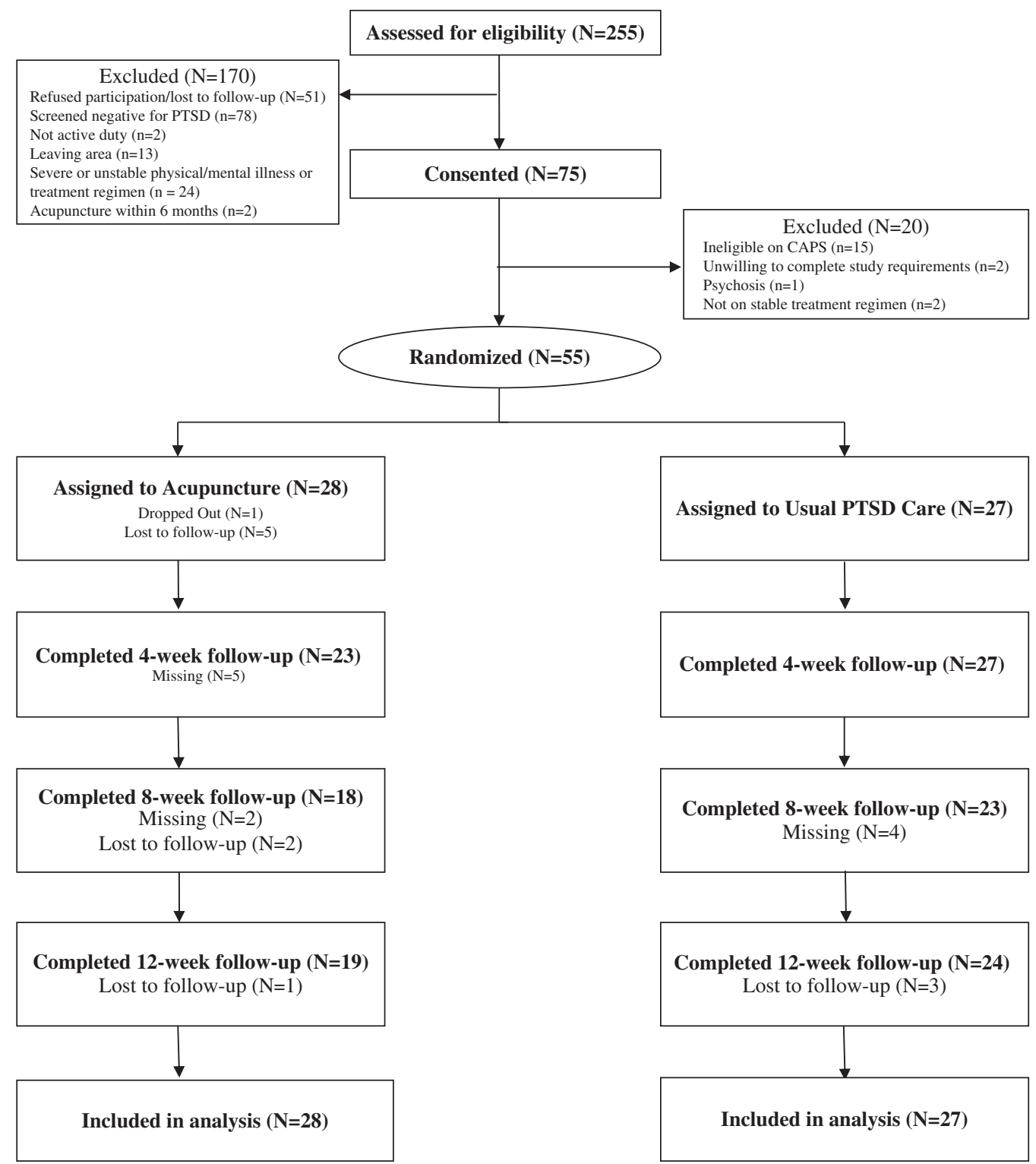

FIGURE 1. CONSORT diagram showing participant flow through screening, enrollment, randomization, treatment, and followup. The drop-out participant completed 1 treatment session and provided 4-week follow-up. Of the participants lost to follow-up during treatment, 2 participants completed 0 treatment sessions, 1 participant completed 3 treatment sessions, 1 participant completed 4 treatment sessions, and 1 participant completed 6 treatment sessions. 
A

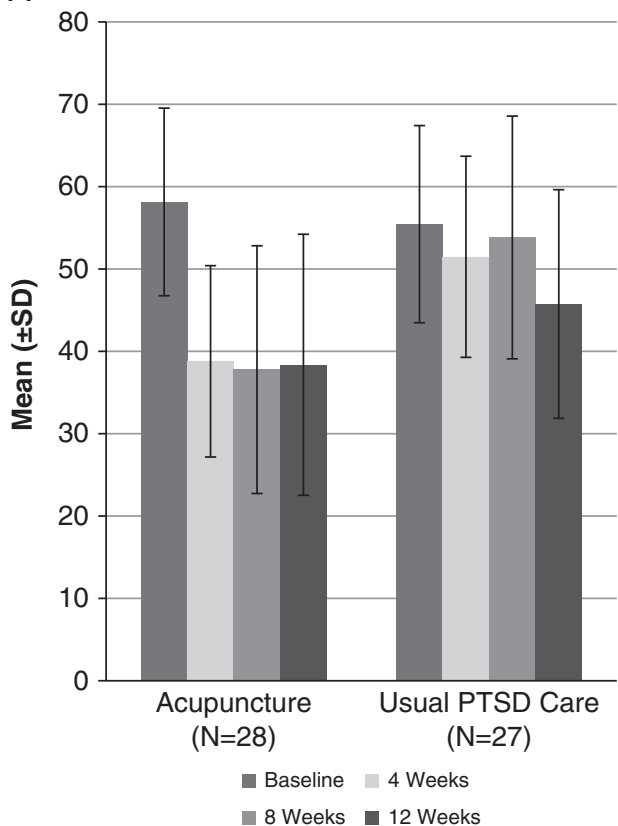

B

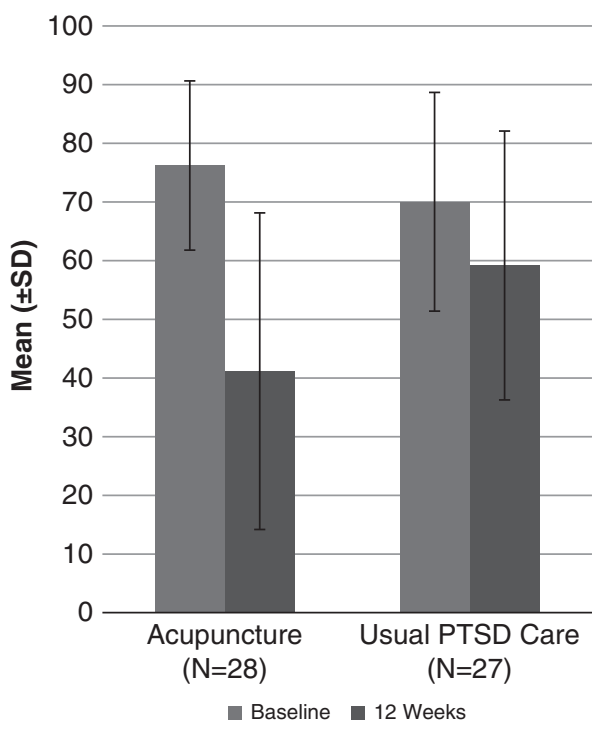

FIGURE 2. PTSD symptom outcomes (acupuncture versus usual PTSD care) on the PCL (primary outcome) (A) and the CAPS (B) for intent-to-treat sample. Treatment $\times$ time comparisons. A, Change in PCL score: acupuncture versus usual PTSD care. B, Change in CAPS score: acupuncture versus usual PTSD care. All $P$-values $<0.0001$ for acupuncture treatment. $P<0.01, P=0.35$, and $P<0.001$ at 4,8 , and 12 weeks, respectively, for PCL in usual PTSD care; $P<0.0001$ for CAPS in usual PTSD care at 12 weeks. CAPS indicates Clinician-administered PTSD Scale; PCL, PTSD Checklist; PTSD, posttraumatic stress disorder.

TABLE 3. Results of Intent-to-treat Sample on Six Mental Health Measures

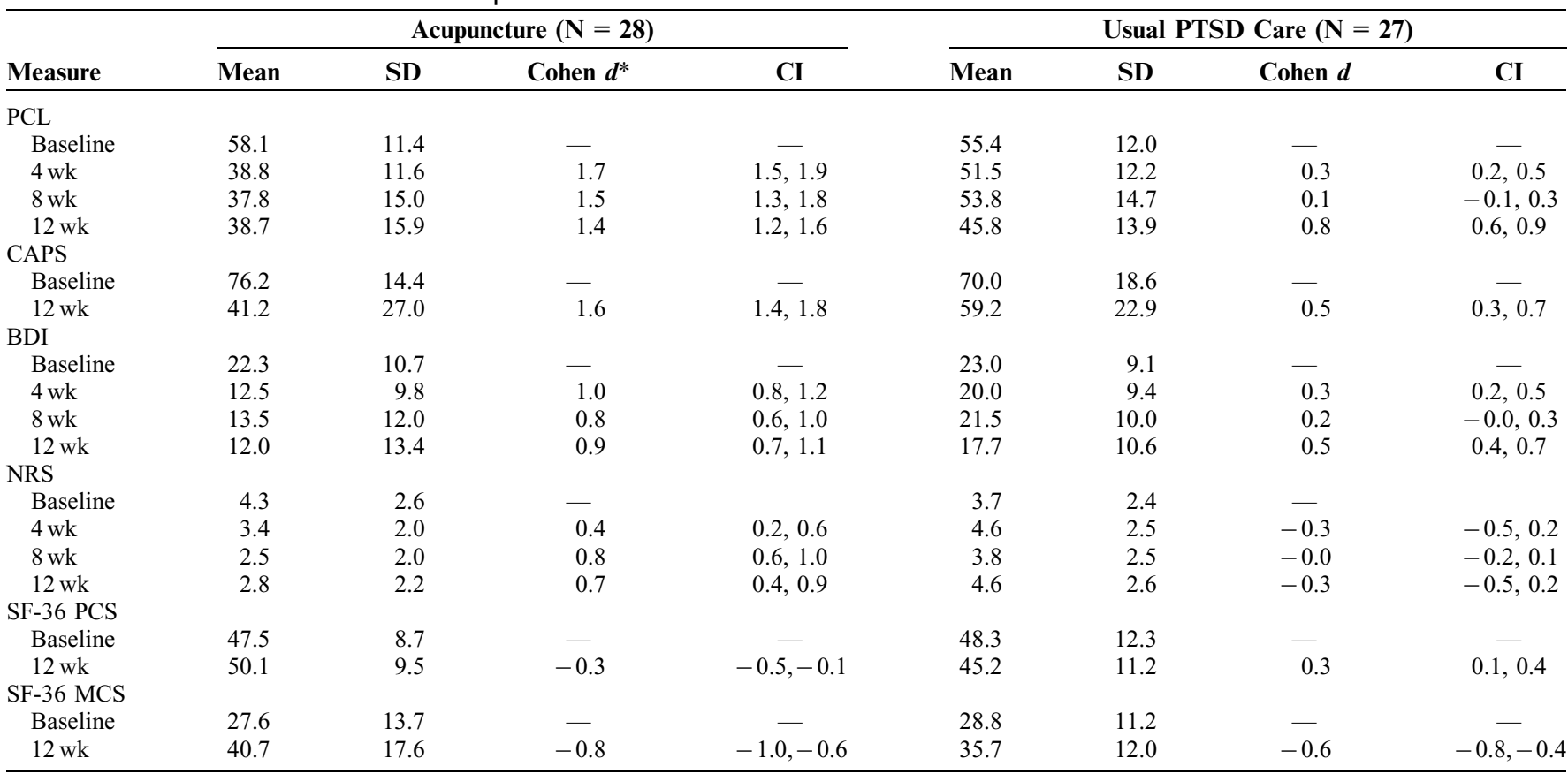

*All effect sizes are pre-post.

BDI indicates total score on the Beck Depression Inventory-II; CAPS, Clinician-administered PTSD Scale total severity score; CI, confidence interval (95\%); NRS, average pain in the past week on the Numeric Rating Scale for Pain; PCL, Posttraumatic Stress Disorder Checklist score; SF36 MCS, Mental Health Component Summary score; SF36 PCS, Physical Component Summary score. 
The least square means were all statistically significant, demonstrating sharper declines in BDI, NRS, PCS, and MCS among participants receiving acupuncture with UPC than among those receiving UPC alone.

The $t$ tests (Table 3) showed a significant drop in the mean BDI-II score in the acupuncture with UPC group from baseline to 4 weeks $\left(22.3 \pm 10.7\right.$ vs. $12.5 \pm 9.8 ; t_{250}=7.7$, $P<0.0001)$ and maintenance of treatment gains at 8 weeks $\left(13.5 \pm 12.0 ; t_{174}=5.7, P<0.0001\right)$ and 12 weeks $(12.0 \pm 13.4$; $\left.t_{160}=11.3, P<0.0001\right)$. In contrast, among those in UPC alone, BDI-II scores exhibited a much smaller change: $t$-values were consistently small at 4 weeks $\left(t_{268}=2.6, P<0.01 ; 23.0 \pm 9.1\right.$ vs. $20.0 \pm 9.4$,$) , at 8$ weeks $\left(t_{232}=1.2, P=0.22 ; 21.5 \pm 10.0\right)$, and at 12 weeks $\left(t_{236}=4.3, P<0.0001 ; 17.7 \pm 10.6\right)$. Likewise, among participants receiving acupuncture with UPC, the mean NRS score of acupuncture participants dropped significantly from baseline to 4 weeks $\left(4.3 \pm 2.6\right.$ vs. $\left.3.4 \pm 2.0 ; t_{252}=3.3, P<0.001\right)$ and maintained the reduction at 8 weeks $\left(2.5 \pm 2.0 ; t_{216}=6.0\right.$, $P<0.0001)$ and 12 weeks $\left(2.8 \pm 2.2 ; t_{211}=4.9, P<0.0001\right)$, whereas in contrast the NRS score increased in the UPC-alone group $\left(3.7 \pm 2.4\right.$ vs. $4.6 \pm 2.5, t_{267}=-2.8, P<0.01 ; 3.8 \pm 2.5$, $t_{237}=-0.35, P=0.74 ; 4.6 \pm 2.6, t_{242}=-2.7, P<0.01$ at 4,8 , and $12 \mathrm{wk}$, respectively). Finally, MSC (mental health functioning composite score) on the SF-36 for those in the acupuncture with UPC group showed significant improvement from baseline to 12 weeks $\left(t_{158}=-6.0, P<0.0001 ; 27.6 \pm 13.7\right.$ vs. $40.7 \pm 17.6)$ as it did for UPC participants $\left(t_{245}=-4.8\right.$, $P<0.0001 ; 28.8 \pm 11.2$ vs. $35.7 \pm 12.0$ ). Acupuncture with UPC participants' PCS (physical health functioning composite score) scores on the SF-36 evidenced modest improvement (47.5 \pm 8.7 vs. $\left.50.1 \pm 9.5 ; t_{180}=-2.1, P<0.05\right)$, compared with the UPCalone group, which reported a slight worsening of PCS scores ( $48.3 \pm 12.3$ vs. $45.2 \pm 11.2 ; t_{253}=2.1, P<0.05$ ).

\section{CONCLUSIONS}

This randomized trial is the first to determine that a brief course of acupuncture designed for safety and ease of implementation is an effective treatment adjunct for PTSD individuals receiving acupuncture with their UPC showed significantly greater improvements than those receiving UPC alone. The benefits associated with acupuncture were wide ranging and robust, yielding improvements in PTSD, depression, and pain severity of sufficient magnitude to benefit global mental and physical health functioning. Participants in both groups received similar levels of nonacupuncture PTSD treatments, suggesting that differing levels of these treatments across groups could not account for the acupuncture benefits observed. Symptom severity effect-sizes were large by usual statistical standards, the onset of the clinical effects occurred early, and improvements persisted for the 8 weeks of follow-up after cessation of acupuncture. For example, PTSD pre-post effect sizes ranged from 1.4 to 1.7 in the acupuncture arm, similar to effect sizes observed in the only other published trial of acupuncture for PTSD ${ }^{25}$ and comparable with those for CBT and exposure, the 2 leading PTSD psychotherapies. ${ }^{5}$

A randomized effectiveness design was used to derive an internally valid and readily generalizable "real-world" estimate of the benefits associated with adding a brief course of acupuncture to the UPC offered to affected military personnel. This design offers readily interpretable information that clinicians and policy-makers can use to improve mental health outcomes and clinical services across a range of treatment settings to include primary and specialty care. Even so, there are limitations associated with the choice of an effectiveness design over a more standard efficacy approach. Because the current study failed to equalize contact time across study arms or to use sham controls, resulting data cannot be used to dismantle placebo and other nonspecific treatment effects or to explore acupuncture's biological underpinnings. To accomplish this, however, would have required a much larger multisite study using more restrictive eligibility criteria and an extended period of follow-up.

Such a study, however, would have been less feasible and more costly, while generating results that would not easily generalize to clinical decision making using acupuncture as an treatment adjunct to more commonly used and widely tested PTSD treatment modalities (eg, selective serotonin reuptake inhibiting agents, prazosin, and empirically validated psychotherapies). Furthermore, the course of manual acupuncture tested in this trial was brief ( 8 sessions delivered over $4 \mathrm{wk}$ ), rendering it relatively affordable, feasible for delivery in an array of health care settings including primary care, and requiring relatively little provider and patient time. Although longer courses of acupuncture using more complicated procedures (eg, electroacupuncture) might have enhanced beneficial effects, our main objective was to test whether a simple and easily implementable procedure could offer clinical settings a feasible, low-cost adjunct to patients' usual medical care.

If the benefits observed in this small trial are confirmed in future studies, acupuncture may offer a unique addition to our current PTSD treatment armamentarium that addresses not only PTSD but other associated features such as pain, depression, loss of function, and health-related quality of life. Acupuncture is neither medication nor psychotherapy and could hold promise for advancing the crucial process of engaging the large fraction of untreated individuals with unmet PTSD-related needs. Future studies of acupuncture for PTSD should continue to evaluate its clinical effectiveness and efficacy when delivered alone and in combination with other treatment modalities. Studies are also necessary to assess the cost-effectiveness of acupuncture delivered in various ways for PTSD as well as acupuncture acceptability among persons with PTSD, particularly those who have not sought PTSD treatment due to stigma or lack of confidence in more standard modalities.

\section{ACKNOWLEDGMENTS}

The authors thank acupuncturists, Alaine D. Duncan, M.Ac., L.Ac., Marjorie G. Shovlin, M.Ac., L.Ac., David Blaiwas, M.Ac. L.Ac., MA, and Kate Walshe, M.Ac., L.Ac. for their extensive effort in support of this project. The authors also thank the Samueli Institute for the technical support.

\section{REFERENCES}

1. Gore KL, Engel CC, Freed MC, et al. Test of a single-item posttraumatic stress disorder screener in a military primary care setting. Gen Hosp Psychiatry. 2000;30:391-397. 
2. Hoge CW, Castro CA, Messer SC, et al. Combat duty in Iraq and Afghanistan, mental health problems, and barriers to care. $N$ Engl J Med. 2004;351:13-22.

3. Institute of Medicine. Treatment of Posttraumatic Stress Disorder: An Assessment of the Evidence. Washington, DC: The National Academies Press; 2008.

4. Stein DJ, Ipser JC, Seedat S. Pharmacotherapy for post traumatic stress disorder (PTSD). Cochrane Database Syst Rev. 2006;1:Cd002795.

5. Bradley R, Greene J, Russ E, et al. A multidimensional meta-analysis of psychotherapy for PTSD. Am J Psychiatry. 2005;162:214-227.

6. Steenkamp MM, Litz BT. Psychotherapy for military-related posttraumatic stress disorder: review of the evidence. Clin Psychol Rev. 2013;33:45-53.

7. Wang PS, Berglund P, Olfson M, et al. Failure and delay in initial treatment contact after first onset of mental disorders in the National Comorbidity Survey Replication. Arch Gen Psychiatry. 2005;62:603-613.

8. Kessler RC. Posttraumatic stress disorder: the burden to the individual and to society. J Clin Psychiatry. 2000;61(suppl 5):4-12; discussion 13-14.

9. Pilkington K, Kirkwood G, Rampes H, et al. Acupuncture for anxiety and anxiety disorders - a systematic literature review. Acupunct Med. 2007;25:1-10.

10. Zhang ZJ, Chen HY, Yip KC, et al. The effectiveness and safety of acupuncture therapy in depressive disorders: systematic review and meta-analysis. J Affect Disord. 2010;124:9-21.

11. Birch S, Hesselink JK, Jonkman FA, et al. Clinical research on acupuncture. Part 1. What have reviews of the efficacy and safety of acupuncture told us so far? J Altern Complement Med. 2004;10: $468-480$.

12. Lee C, Crawford C, Wallerstedt D, et al. The effectiveness of acupuncture research across components of the trauma spectrum response (tsr): a systematic review of reviews. Syst Rev. 2012;1:45-63.

13. Vickers AJ, Cronin AM, Maschino AC, et al. Acupuncture for chronic pain: individual patient data meta-analysis. Arch Intern Med. 2012;172: 1444-1453.

14. Haker E, Egekvist H, Bjerring P. Effect of sensory stimulation (acupuncture) on sympathetic and parasympathetic activities in healthy subjects. J Auton Nerv Syst. 2000;79:52-59.

15. Hui KK, Liu J, Makris N, et al. Acupuncture modulates the limbic system and subcortical gray structures of the human brain: evidence from fMRI studies in normal subjects. Hum Brain Mapp. 2000;9:13-25.

16. Kong J, Ma L, Gollub RL, et al. A pilot study of functional magnetic resonance imaging of the brain during manual and electroacupuncture stimulation of acupuncture point (LI-4 Hegu) in normal subjects reveals differential brain activation between methods. J Altern Complement Med. 2002;8:411-419.

17. Cabyoglu MT, Ergene N, Tan U. The mechanism of acupuncture and clinical applications. Int J Neurosci. 2006;116:115-125.

18. Cannistraro PA, Rauch SL. Neural circuitry of anxiety: evidence from structural and functional neuroimaging studies. Psychopharmacol Bull. 2003;37:8-25.

19. Sher L. The role of the endogenous opioid system in the pathogenesis of anxiety disorders. Med Hypotheses. 1998;50:473-474.

20. Kaptchuk TJ. Acupuncture: theory, efficacy, and practice. Ann Intern Med. 2002;136:374-383.

21. Staud R, Price DD. Mechanisms of acupuncture analgesia for clinical and experimental pain. Expert Rev Neurother. 2006;6:661-667.
22. Sugiyama Y, Xue YX, Mano T. Transient increase in human muscle sympathetic nerve activity during manual acupuncture. Jpn J Physiol. 1995;45:337-345.

23. Knardahl S, Elam M, Olausson B, et al. Sympathetic nerve activity after acupuncture in humans. Pain. 1998;75:19-25.

24. Kim YD, Heo I, Shin BC, et al. Acupuncture for posttraumatic stress disorder: a systematic review of randomized controlled trials and prospective clinical trials. Evid Based Complement Altern Med. 2013; 2013:615857. [Epub February 6].

25. Hollifield M, Sinclair-Lian N, Warner TD, et al. Acupuncture for posttraumatic stress disorder: a randomized controlled pilot trial. J Nerv Ment Dis. 2007;195:504-513.

26. Zhang H, Ran L, Yuan X, et al. Clinical observation on acupuncture and moxibustion in treating post traumatic stress disorder after 5.12 earthquake. J Chengdu Univ Tradit Chin Med. 2010;33.

27. Zhang H, Yuan C, Ran L, et al. RCT research of different acupuncture therapies in treating posttraumatic stress disorder after Wenchuan ' 5.12 ' earthquake. China J Tradit Chin Med Pharm. 2010;25:1505-1510.

28. Zhang Y, Feng B, Xie JP, et al. Clinical study on treatment of the earthquake-caused post-traumatic stress disorder by cognitivebehavior therapy and acupoint stimulation. J Tradit Chin Med. 2011;31:60-63.

29. Vincent C, Lewith G. Placebo controls for acupuncture studies. J R Soc Med. 1995;88:199-202.

30. Weathers FW, Litz BT, Herman JA, et al. The PTSD Checklist (PCL): Reliability, Validity, and Diagnostic Utility. Annual Convention of the International Society for Traumatic Stress Studies. Paper from proceedings of November 1993 International Society for Traumatic Stress Studies in San Antonio TX. 1993.

31. Blake DD, Weathers FW, Nagy LM, et al. The development of a Clinician-Administered PTSD Scale. J Trauma Stress. 1995;8:75-90.

32. Cleeland CS, Ryan KM. Pain assessment: global use of the Brief Pain Inventory. Ann Acad Med Singapore. 1994;23:129-138.

33. Management of Post-Traumatic Stress Working Group. $V A / D o D$ Clinical Practice Guideline for the Management of Post-Traumatic Stress. Washington, DC: Department of Veterans Affairs and Department of Defense; 2012.

34. Calohan J, Peterson K, Peskind ER, et al. Prazosin treatment of trauma nightmares and sleep disturbance in soldiers deployed in Iraq. J Trauma Stress. 2010;23:645-648.

35. Blake DD, Weathers FW, Nagy LM, et al. The development of the Clinician-Administered PTSD Scale. J Trauma Stress. 1995;8:75-90.

36. Beck AT, Steer RA, Ball R, et al. Comparison of Beck Depression Inventories-IA and -II in psychiatric outpatients. J Pers Assess. 1996;67:588-597.

37. Ware J, Snow KK, Kosiniski M, et al. SF-36 Health Survey: Manual and Interpretation Guide. Boston, MA: Quzlity Metric Inc. and The Health Assessment Lab; 1993.

38. Littell R, Woodward SH, Young HE, et al. SAS System for Mixed Models. Cary, NC: SAS Institute Inc; 1996.

39. Shafer JL. Analysis of Incomplete Multivariate Data. Boca Raton, FL: Chapman and Hall/CRC; 1997.

40. Robey RR. Reporting point and interval estimates of effect-size for planned contrasts: fixed within effect analyses of variance. J Fluency Disord. 2004;29:307-341. 